\title{
$\underline{P-151}$
}

\section{Subcritical Water Extraction (SWE) OF Eurycomanone and Eurycomanol from Eurycoma Longifolia Jack Roots}

\author{
Mohamad Firdhaus Ramli, Norashikin Saim and Rohaya Ahmad \\ Faculty of Applied Science, Universiti Teknologi Mara, $40450 \quad$ Shah Alam, Selangor; \\ E-mail: noras691@salam.uitm.edu.my
}

As an alternative to tradition extraction method, the ability of subcritical water extraction(SWE) to extract bioactive compounds, eurycomanone and eurycomanol from Eurycoma longifolia Jack was investigated. The operational parameters of SWE, such as temperature, pressure and static extraction time were optimized using central composite design (CCD). By applying multiple regression analysis on the experiment data obtained, the result of the CCD was fitted with a second order quadratic equation.. The results showed that the only significant variable which affects the extraction efficiency of both compounds is the extraction temperature. The optimal conditions of SWE were extraction temperature of $104^{\circ} \mathrm{C}$, extraction pressure of $1700 \mathrm{psi}$, using one extraction cycle with a static extraction time of $14 \mathrm{~min}$. The yield of these bioactive compounds extracted using SWE was compared with that obtained by Soxhlet extraction using methanol as the extracting solvent.

Keywords: SWE, CCD, eurycomanone, eurcomanol, Eurycoma logifolia Jack. 